\title{
Computing Convolutions by Reciprocal Search
}

\author{
Leonidas J. Guibas ${ }^{1}$ and Raimund Seidel ${ }^{2 *}$ \\ ${ }^{1}$ Stanford University and DEC/SRC, 130 Lytton, Palo Alto, CA 94301, USA \\ ${ }^{2}$ Computer Science Department, Cornell University, Ithaca, NY 14853, USA \\ Communicated by David Dobkin
}

\begin{abstract}
In this paper we show how certain geometric convolution operations can be computed efficiently. Here "efficiently" means that our algorithms have running time proportional to the input size plus the output size. Our convolution algorithms rely on nev. optimal solutions for certain reciprocal search problems, such as finding intersections between "blue" and "green" intervals, and overlaying convex planar subdivisions.
\end{abstract}

\section{Introduction}

A reciprocal search problem can be informally defined as follows: Let $B$ be a set of $m$ "blue" objects, and $G$ be a set of $n$ "green" objects. Among all the objects some relation $\rho$ is defined. Find all pairs of differently colored, related objects, i.e., find all $(b, g)$, with $b \in B$ and $g \in G$, such that $b \rho g$.

Reciprocal search problems arise frequently in computational geometry. Typically, the objects involved are points, line segments, rectangles, or other figures with simple descriptions, and the relation $\rho$ might be something like "having nonempty intersection." A special feature of this kind of problem is the large possible variation in the output size. No blue-green object pair might be in the relation, or all of the $m n$ pairs might be in the relation. Thus the running time of a good algorithm for such a problem should be sensitive to the output size, which we denote by $K$.

In a standard computational geometry approach a reciprocal search problem is usually reduced to a one-sided search problem. One of the sets, say $B$, is stored

* This research was done while on leave from Comell at DEC/SRC. Present address: IBM Almaden Research Center, K53/801, 650 Harry Road, San Jose, CA 95120-6099, USA. 
in some appropriate data structure that can efficiently answer queries of the form "for some object $x$, which $b \in B$ satisfy $x \rho b$." Subsequently, such a query is performed for each green object in $G$. Such an approach can have certain shortcomings. Possible symmetry of the relation $\rho$ remains unexploited, and structure useful to our problem present in the blue set $B$ might be lost in the data structure. Similarly, any structure in $G$ is ignored, since each of its elements is used for a query individually and out of context. Finally, it is somewhat displeasing that $B$ and $G$ play interchangeable roles in the problem definition but very different roles in the problem solution.

In this paper we give solutions for two important instances of reciprocal search problems. One has to do with intersecting contiguous chains of intervals, and the other with intersecting convex subdivisions. In each case we manage to exploit the symmetry or "reciprocality" of the problem. This enables us to obtain optimal $O(m+n+K)$ algorithms, i.e. algorithms which run in time proportional to the size of their inputs plus their output. These algorithms in turn yield optimal algorithms for a calculus of tracings, a new framework for computational geometry first introduced by Guibas et al. in [5]. The operation of convolving polygonal tracings in two dimensions, as well as that of convolving a certain class of polyhedral tracings in three space involve exactly the subproblems mentioned above. Thus reciprocal search provides us with optimal algorithms for these important convolution problems.

Section 2 outlines our solution for the reciprocal search problem of finding all intersections between two contiguous chains of intervals. In Section 3 we outline a solution for constructing the intersections between the elements of two planar convex subdivisions. In Sections 4 and 5 we apply our results to the calculus of tracings. In this application some subtle issues arise about the computer representation of tracings. Since these subtleties are not the primary focus of this work, we have refrained from discussing them at length in the main body of the paper. However, we have included in the appendix a short discussion of the main issues involved.

\section{Intersecting Contiguous Chains of Intervals}

We are interested in the following reciprocal search problem: given a finite set $B$ of "blue" arcs and a finite set $G$ of "green" arcs on the unit circle we want to report all intersections between blue and green objects. To simplify our presentation we consider what is in essence an equivalent problem, namely that of finding intersections of intervals on the real line: for a set $B=\left\{b_{i} \mid 1 \leq i \leq m\right\}$ of $m$ (topologically) closed "blue" intervals and a set $G=\left\{g_{j} \mid 1 \leq j \leq n\right\}$ of $n$ closed "green" intervals report all intersections between blue and green intervals, all containments of green (blue) endpoints in blue (green) intervals, and all coincidences of green and blue endpoints.

It is not difficult to devise an algorithm that first sorts the endpoints of both $B$ and $G$ and then reports all the desired intersections in one scan over the sorted list. Such an algorith could be implemented to run in $O((m+n) \log (m+n)+K)$ time, where $K$ is the number of intersections reported. If $K$ is small, the running 
time of this algorithm is dominated by the $(m+n) \log (m+n)$ term required for sorting. It is also not hard to show that this is a lower bound to this problem in the general setting. However, in our application for tracings the input sets $B$ and $G$ have additional structure: they are both contiguous, that is, for $1 \leq i<m$ the intervals $b_{i}$ and $b_{i+1}$ have a common endpoint and similarly for $1 \leq j<n$ the intervals $g_{j}$ and $g_{j+1}$ share an endpoint. We will show that with this input restriction we can report all intersections in time $O(n+m+K)$.

First note that it suffices to deal with the problem of reporting endpoint-interval containments since interval-interval intersections and endpoint-endpoint coincidences can be inferred from these containments. Consider the case of reporting the blue intervals that contain a given green endpoint $t$.

It is convenient to view this as a problem in the plane. For each interval $b_{i}$ let $b_{i}^{\prime}$ be the segment $b_{i} \times\{i\}$ on the horizontal line $y=i$ directly above $b_{i}$. Clearly, $t$ is contained in $b_{i}$ iff the vertical line $T=\{(x, y) \mid x=t\}$ intersects $b_{i}^{\prime}$. Thus we want to be able to answer queries asking which $b_{i}^{\prime \prime}$ 's intersect a given vertical line $T$. Chazelle [2] introduced a data structure, called hive-graph, that allows such queries to be answered in time $O(k+\log m)$, where $k$ is the answer size.

A hive-graph is basically a decomposition of the plane into rectangles (some of which may be unbounded) that are aligned with the axes and have the following properties: (1) the vertical sides of the rectangles only lie on lines that contain an endpoint of some $b_{i}^{\prime}$, (2) each horizontal side is contained in exactly one $b_{i}^{\prime}$, and (3) every rectangle has at most two rectangles directly above it (see Fig. 1). In general, such a hive-graph can be built in $O(m \log m)$ time using $O(m)$ space using a sweep algorithm. However, in our case, where the $b_{i}^{\prime \prime}$ 's are already naturally ordered in the vertical direction and each pair of segments $b_{i}^{\prime}$ and $b_{i+1}^{\prime}$ has an endpoint on a common vertical line, such a hive-graph can be built in $O(m)$ time.

Call a rectangle in the hive-graph that is unbounded from below a bottom rectangle. Note that the bottom rectangles are naturally ordered horizontally.

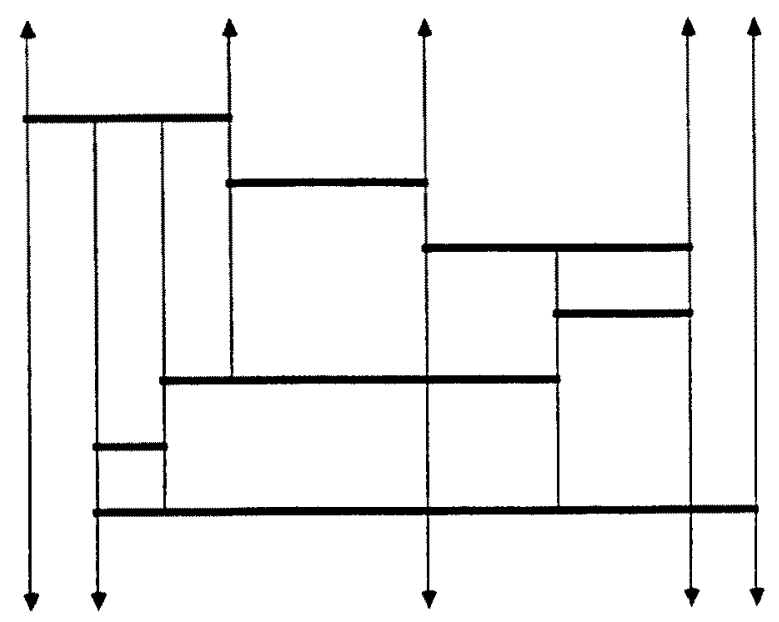

Fig. 1. Hive-graph. 
A query can be answered as follows: first determine which of the bottom rectangles intersects the vertical line $T$ in question; starting from there, find all other rectangles that intersect $T$ in vertical order. For each discovered rectangle (except for the initial one) report the segment $b_{i}^{\prime}$ that contains its lower horizontal side. These segments are exactly the ones that intersect $T$.

The first step can be performed at logarithmic cost via binary search. The second step can be performed at constant cost per rectangle found as every rectangle has at most two rectangles directly above it. Thus the overall cost for performing such a query for each green endpoint is $O(m)$, for setting up the hive-graph, $O(n \log m)$ for performing the first steps, and $O(k)$ for reporting, where $k$ is the total number of blue intervals reported.

We would like to eliminate the $O(n \log m)$ factor. This can be achieved as follows: recall that the green intervals are also contiguous, i.e., $g_{j}$ and $g_{j+1}$ share an endpoint. Treat the green intervals in order of increasing $j$. Let $a$ and $b$ be the two endpoints of $g_{j+1}$. Without loss of generality assume that $a$ is also an endpoint of $g_{i}$. Having processed $g_{i}$ earlier means that a query for $a$ has been performed already. Thus we know the bottom rectangle $R$ that intersects the vertical line through $a$. To find the corresponding rectangle for $b$ we just search sequentially through the sorted list of bottom rectangles starting at $R$. Note that the vertical rectangle sides crossed by this sequential search stem from endpoints of blue intervals by the properties of the hive-graph. Thus the number of such crossings must not be greater than the number of blue endpoints contained in the green interval $g_{j+1}$. It follows that finding the initial bottom rectangles for all the green endpoints can be performed in time $O\left(\log m+k^{\prime}\right)$, where $k^{\prime}$ is the number of blue-endpoint-green-interval containments, and the $\log m$ factor stems from treating the first endpoint of $g_{1}$. Therefore the time necessary to perform the queries for all green endpoints is $O\left(m+k+k^{\prime}\right)$.

Applying the same method to the symmetric case of blue-endpoint-greeninterval containments yields the desired optimal result:

Lemma 1. For a continguous set of $m$ blue intervals and a contiguous set of $n$ green intervals all blue-endpoint-green-interval and green-endpoint-blue-interval containments can be found in time $O(m+n+K)$, where $K$ is the number of containments reported.

\section{Overlaying Planar Convex Subdivisions}

A convex subdivision of the plane is a partition of the plane into a finite number of (relatively) open convex sets. It is not hard to see that these convex sets can be only of the following three types; a set can be a two-dimensional region, i.e., it is an open convex polygon (possibly unbounded); a set can be a one-dimensional edge, i.e., a (possibly unbounded) interval on some straight line; and a set can be a zero-dimensional vertex. Regions are separated by edges and vertices form endpoints of edges. Examples of convex subdivisions are Voronoi diagrams and straight-line arrangements. 


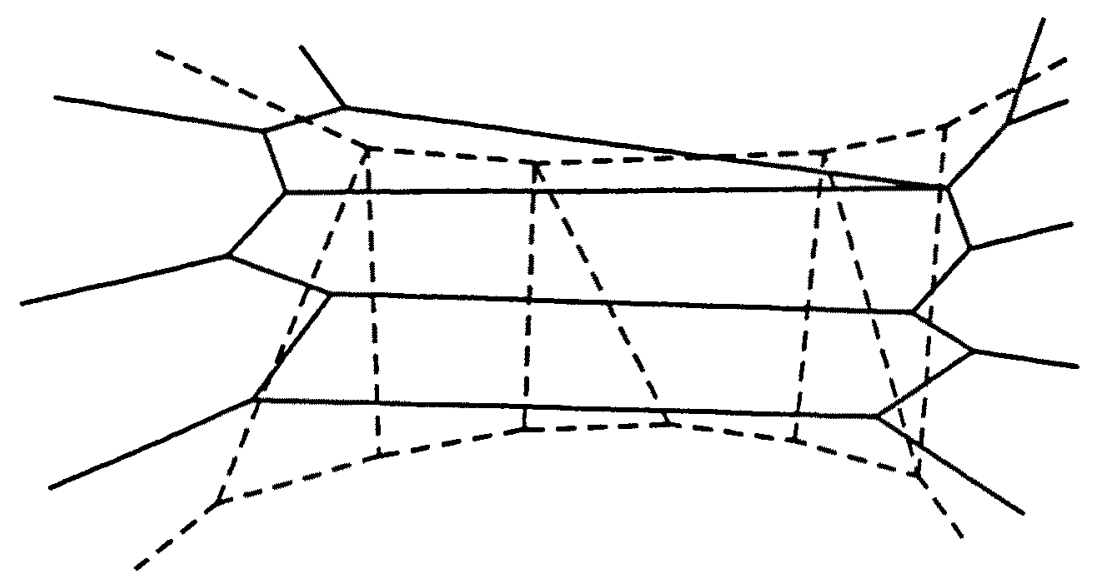

Fig. 2. Overlay of two subdivisions.

From any two planar convex subdivisions $\mathscr{B}$ and $\mathscr{G}$ one can form a new convex subdivision which we call the overlay of $\mathscr{B}$ and $\mathscr{G}$. It consists of all nonempty sets of the form $B \cap G$ with $B \in \mathscr{B}$ and $G \in \mathscr{G}$. We are interested in computing the overlay of two convex subdivisions efficiently.

First we have to settle the question of representation. We assume that convex subdivisions are represented by some suitable planar graph data structure (for instance the quad edge structure [6]), appropriately augmented to be able to represent unbounded edges and regions. We also have to specify what we mean by "efficient computation." Note that the overlay of two convex subdivisions $\mathscr{B}$ and $\mathscr{G}$ of size $m$ and $n$, respectively, can consist of as many as $O(m n)$ sets, as alluded to in Fig. 2. On the other hand, it is not hard to exhibit examples where the overlay consists of only $O(m+n)$ sets. It is thus desirable that the running time of an overlay construction algorithm also depends on the size of the produced convex subdivision. In the following we let $K$ denote the size of the produced overlay subdivision.

From now on we will designate the subdivision $\mathscr{B}$ and its parts as blue and the subdivision $\mathscr{G}$ and its parts as green. For ease of presentation we will assume that $\mathscr{B}$ and $\mathscr{G}$ are in "nondegenerate" position, i.e., they both have no vertical edges, no blue and green vertices coincide, and no blue (green) vertex lies on a green (blue) edge.

The obvious approach for solving the overlay problem is the plane sweep paradigm: sweep a vertical line from left to right over the two subdivisions and maintain the invariant that the part of the overlay that is totally to the left of the sweeping line has been correctly constructed. In its most natural form such a sweeping algorithm takes time $O((m+n+K) \log (m+n))$ (see, e.g., [1]). By exploiting convexity and changing the sweeping invariant a bit (in the sense that parts of the overlay to the right of the sweeping line must also be computed) one can design a more sophisticated algorithm with running time $O((m+n) \log (m+n)+K)[7]$. We will use the idea of a "topological line" 
sweep as proposed by Edelsbrunner and Guibas [3] to construct the overlay in optimal $O(m+n+K)$ time.

The topoligical line sweep is a generalization of a straight line sweep. A line is swept over the plane from left to right and at all times the invariant is maintained that everything to the left of the line has been correctly computed already. However, the sweeping line is not a straight vertical line. It is allowed to bulge to a certain extent. In order to quantify the legal amount of bulging, and also for the purpose of dealing with such a line algorithmically, we must give a combinatorial characterization of what constitutes a correct sweeping line. We do this by identifying the line by the sequence of subdivision edges that intersect the line. We say an edge $a$ of a subdivision lies immediately above an edge $b$ iff there is a region $R$ in the subdivision such that $a$ is a top edge of $R$ and $b$ is a bottom edge of $R$, i.e., $a$ and $b$ are edges bounding $R$ from above and below, respectively. A sequence of edges $e_{1}, e_{2}, \ldots, e_{s}$ constitutes a cut of a subdivision $\mathscr{Z}$ iff $e_{1}$ is a bottom edge of the top region of $\mathscr{X}, e_{s}$ is a top edge of the bottom region of $\mathscr{X}$, and $e_{i}$ lies immediately above $e_{i+1}$ for $1 \leq i<s$. (Note that because of convexity and the assumption of no vertical edges all the notions involving "top" and "bottom" are well defined. Moreover, the top and bottom regions of $\mathscr{Z}$ are unique.) A line $L$ is said to be a valid sweep line for a subdivision $\mathscr{X}$ iff the sequence of edges of $\mathscr{X}$ that intersect $L$ forms a cut of $\mathscr{X}$ (see Fig. 3). Note that for every cut $C$ of $\mathscr{X}$ there is a valid sweep line $L_{C}$. One can define a partial order on the cuts of a subdivision $\mathscr{Z}$ by saying that a cut $C$ is to the left of a cut $C^{\prime}$ iff the set of vertices of $\mathscr{X}$ to the left of $L_{C}$ is a subset of the set of vertices to the left of $L_{C}$. There is a unique leftmost cut, the one with no vertices to the left of $L_{C}$, and a unique rightmost cut, the one with all vertices to the left of $L_{C}$. Sweeping a subdivision $\mathscr{X}$ entails starting with the leftmost cut $C_{0}$ and then going through a sequence of cuts $C_{i}$ until the rightmost cut $C_{t}$ is reached. Moreover,

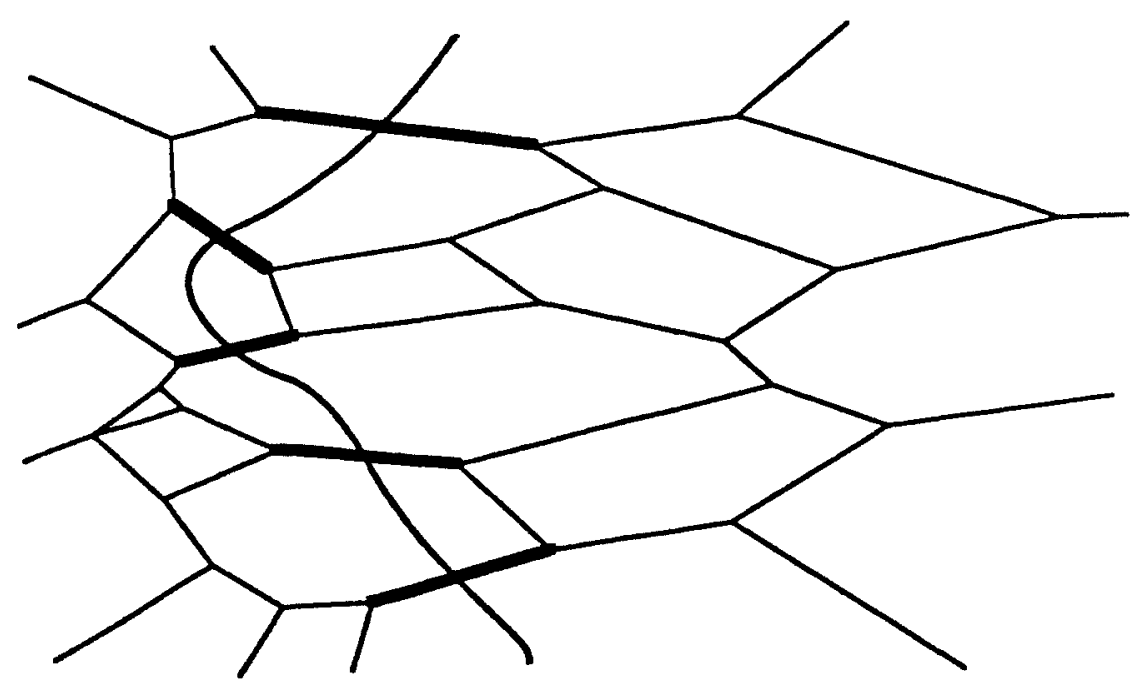

Fig. 3. A cut and a sweep line. 


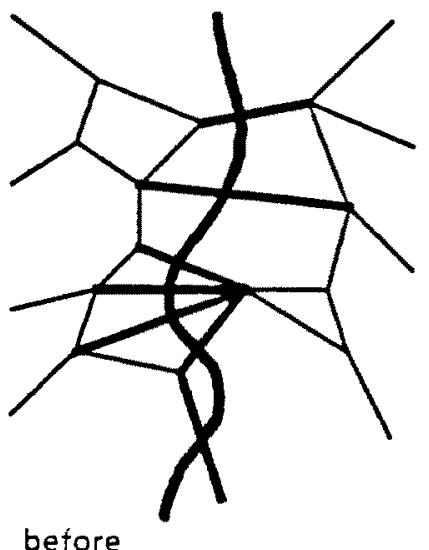

before

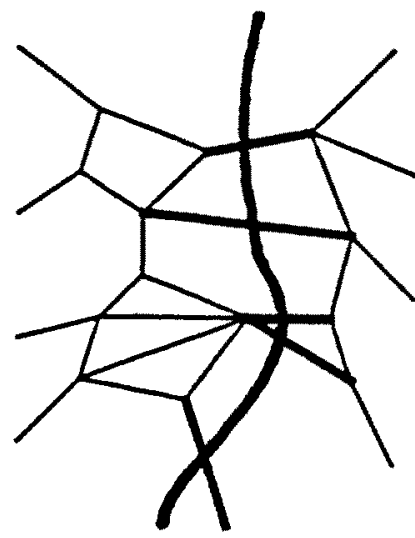

after

Fig. 4. Sweeping over a vertex.

for all $i, 0 \leq i<t, C_{i}$ must be left of $C_{i+1}$, and the difference between $C_{i}$ and $C_{i+1}$ should be "small," i.e., one more vertex is to the left of $C_{i+1}$ than to the left of $C_{i}$.

For a cut $C_{i}$ in a sweep what can be the next cut $C_{i+1}$ ? Which vertex of the subdivision can be swept over next? It is easy to verify that any vertex $v$ for which all left-edges (i.e., edges incident to and to the left of $v$ ) lie in the current cut $C_{i}$ can be chosen for this purpose. The new cut $C_{i+1}$ is then the same as $C_{i}$ except that the left-edges of $v$ are replaced by the right-edges of $v$ in their natural vertical order (see Fig. 4). We now know how a sweep can proceed. Is it possible that a sweep can get stuck? Is it possible that there is no vertex whose left-edges are in the current cut (provided, of course, we are not dealing with the rightmost cut)? The answer to this question is negative. Consider the leftmost of the vertices to the right of the current cut. Obviously all its left-edges must be in the cut. It is worth pointing out that the straight line sweep relies on this fact and always chooses as the next vertex to sweep over the one that is leftmost among the vertices to the right of the sweeping line. Note that this is a global condition. The main advantage of the topological line sweep lies in the fact that it uses instead the purely local conditions of "all left-edges in the current cut" for advancing the sweep, thus saving a logarithmic factor in the running time.

For the purpose of constructing the overlay $\mathscr{X}$ of two subdivisions $\mathscr{B}$ and $\mathscr{G}$ the sweeping approach will be applied as follows. Conceptually we will sweep over the result subdivision $\mathscr{X}$. Of course we do not know $\mathscr{E}$ in advance. However, we know that it is made up of $\mathscr{B}$ and $\mathscr{G}$ and we will exploit the fact that every cut of $\mathscr{X}$ is a cut of $\mathscr{B}$ merged with a cut of $\mathscr{G}$. Moreover, we will maintain the invariant that all parts of $\mathscr{X}$ that are completely to the left of the current sweep line have been correctly identified and constructed.

To start the sweep we have to construct the leftmost cut of $\mathscr{Z}$. Note that the leftmost cut of any convex subdivision consists of the edges which are unbounded to the left sorted according to their slope. Thus the leftmost cut of $\mathscr{X}$ can be formed from the leftmost cuts of $\mathscr{B}$ and $\mathscr{G}$ by a simple merge operation in linear 
time. Throughout the sweep we will augment the sequence of edges in the current cut with a sentinel edge on each end. These sentinel edges are taken to be both green and blue and they are assumed to be unbounded to the right.

In the resulting subdivision $\mathscr{X}$ there are really three types of vertices: (i) vertices that are formed by the intersection of blue and green edges, (ii) vertices of the blue subdivision, and (iii) vertices of the green subdivision. Note that the type (i) vertices are not known in advance but are discovered on the fly. We have three rules for advancing the sweeping line, according to the type of the vertex we are currently sweeping over.

Rule 1. If in the current cut there are two consecutive edges $e_{i}$ and $e_{i+1}$ of different color which intersect so that their vertical order to the right of their intersection point is not reflected by their order in the current cut, then

interchange the two edges in the cut, make their intersection point $p$, and the segments of the edges to the left of $p$ part of $\mathscr{X}$, etc.

Rule 2. If $v$ is a safe blue vertex whose left-edges form a contiguous subsequence of the current cut, then

delete these edges from the cut, and

insert instead in their natural vertical order the right-edges of $v$.

Rule 3. Same as Rule 2 but interchange blue and green.

We owe a definition of what it means for a blue vertex $v$ to be safe. Let $e$ and $e^{\prime}$ be the green edges immediately above and below $v$, respectively. Vertex $v$ is $s a f e$ iff neither $e$ nor $e^{\prime}$ is to the right of the current cut. Safety of green vertices is defined symmetrically. Note that once a vertex is safe during a sweep it stays safe.

We want to argue the correctness of Rules 1-3. Correctness in this context means that the sweeping invariant "all parts of $\mathscr{X}$ to the left of the sweeping line have been identified correctly" is maintained. Consider first Rule 1: let $e_{i}$ and $e_{i+1}$ be edges satisfying the conditions of Rule 1 . Without loss of generality let $e_{i}$ be blue and $e_{i+1}$ be green. Let $L$ be some sweep line corresponding to the current cut and let $q_{i}$ and $q_{i+1}$ be the intersection points of $L$ with $e_{i}$ and $L$ with $e_{i+1}$, respectively. Finally, let $p$ be the intersection of $e_{i}$ and $e_{i+1}$ (see Fig. 5). We need to show that no green edge can intersect $e_{i}$ between $q_{i}$ and $p$. By the conditions of Rule 1 no green edge intersects $L$ between $q_{i}$ and $q_{i+1}$. Similarly, no green edge intersects $e_{i+1}$. Let $p^{\prime}$ be a point on $e_{i}$ to the left of, but arbitrarily close to, $p$. The points $p^{\prime}$ and $q_{i}$ must lie in the same region of the green subdivision; they can be joined by a path running arbitrarily close along $L$ and $e_{i+1}$ that does not cross any green edges. By convexity the straight line segment joining $p^{\prime}$ and $q_{i}$ (which is a subset of $e_{i}$ ) must be contained entirely in one green region. Thus no green edge can intersect this segment and hence no green edge can intersect 


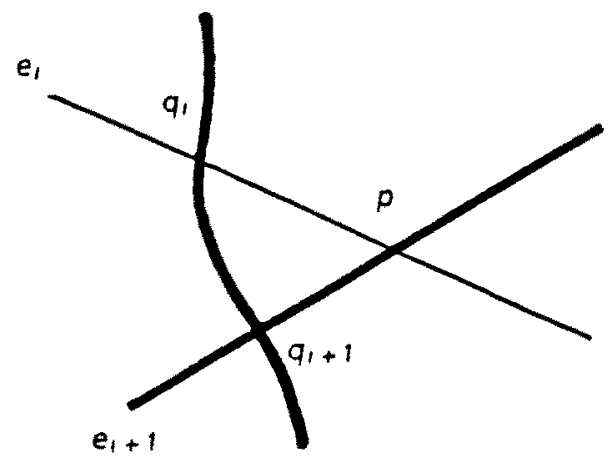

Fig. 5. Correctness of Rule 1.

$e_{i}$ between $q_{i}$ and $p$. By the same reasoning no blue edge can intersect $e_{i+1}$ between $q_{i+1}$ and $p$.

Next consider the correctness of Rule 2. Let $v$ be a blue vertex satisfying the conditions of Rule 2. Let $G$ be the region of the green subdivision that contains $v$ and let $e$ and $e^{\prime}$ be the green edges bounding $G$ that lie directly above and below $v$, respectively. By the safety of $v$ the edges $e$ and $e^{\prime}$ are either on or totally to the left of the current cut. Thus all bounding edges of $G$ to the left of $e$ and $e^{\prime}$ are completely to the left of the current cut and therefore, by the sweeping invariant, all intersections of these edges with blue edges have been correctly discovered already. For any left-edge of $v$ let $\bar{e}$ be the last (rightmost) intersecting green edge. Clearly, $\bar{e}$ must be a bounding edge of $G$ that it not to the right of $e$ or $e^{\prime}$ which means $\bar{e}$ is either on or to the left of the current cut. If $\bar{e}$ is to the left of the current cut then all intersections with blue edges must have been discovered already because of the arguments above. If $\bar{e}$ is part of the current cut then all intersections with left-edges of $v$ must have been discovered already because of the contiguity assumption for the left-edges of $v$ in the current cut. Thus it is safe to sweep over vertex $v$ and Rule 2 (and hence also the symmetrical Rule 3 ) is indeed correct.

In order to be able to apply Rules 2 and 3 we need to know which vertices are safe. The condition for safety appears to be easy to ascertain. However, it turns out to be hard to maintain the set of all safe vertices at all times at reasonable cost. We circumvent this problem by maintaining just a subset of the safe vertices. For this purpose we have the following two rules.

Rule 4. In the current cut let $e_{i}$ and $e_{k}$ be two blue edges with $e_{j}$ green for all $j, i<j<k$, and let $B$ be the blue region between $e_{i}$ and $e_{k}$. If the right endpoints of both $e_{i}$ and $e_{k}$ lie to the right of the right endpoint $r$ of the edge $e_{l}$, for $l=i+1$ or $l=k-1$, then

declare $r$ to be safe;

declare safe all green vertices to the left of $r$ that have not been declared safe 
so far and that can be reached by an $x$-monotone green edge path from $r$ that stays entirely within the blue region $B$.

Rule 5. Same as Rule 4, but interchange blue and green.

It should be clear that Rules 4 and 5 are indeed correct in the sense that they do not declare vertices safe which in reality are not. Also note that all the vertices declared safe by (say) Rule 4 can be easily identified by starting a depth-first search through the left-edges of $r$ that backtracks whenever $e_{i}$ or $e_{k}$ would be crossed, a cut edge $e_{j}$, with $i<j<k$, is reached, or an already safe vertex is encountered. It remains to be shown that these rules are sufficiently encompassing, that it is impossible that Rules 1-3 cannot be applied because no appropriate safe vertex has been discovered via Rules 4 and 5 .

So let us assume that Rule 1 cannot be applied (and we are not dealing with the rightmost cut). Call a (nonsentinel) edge $e_{i}$ in the current cut essential iff $e_{i-1}$ or $e_{i+1}$ has a different color than $e_{i}$. Let $r$ be the leftmost right endpoint of the essential edges in the current cut. Without loss of generality assume $r$ is green. Rule 4 is obviously applicable to $r$. Let $v$ be the leftmost green vertex that can be reached by an $x$-monotone edge path as specified in Rule 4 . We claim that the left-edges of $v$ form a contiguous subsequence of the current cut and thus Rule 2 is applicable. Since $v$ is leftmost the only way that left-edges of $v$ might not lie in the current cut is for them to intersect $e_{i}$ or $e_{k}$ (as in Rule 4). But this would contradict the leftmost condition for $r$ (see Fig. 6). Thus all left-edges of $v$ lie in the current cut. They have to be contiguous since otherwise Rule 1 would still be applicable, contrary to assumption.

In order to use Rules 1-5 efficiently in an algorithm one needs to maintain the following information: a double-linked list of the edges in the current cut; for each of these edges a pointer to the next and to the previous edge of the

some essential edge must have right endpoint in shaded area

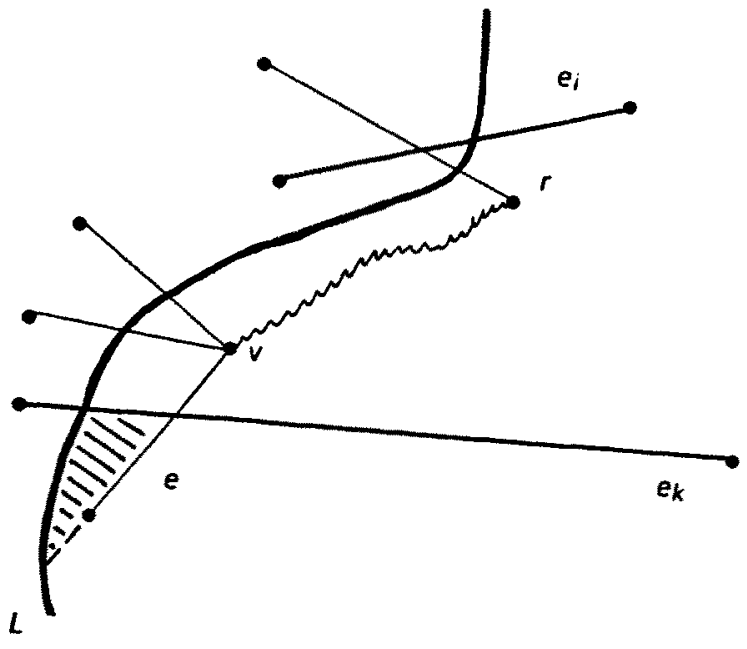

Fig. 6. Applicability of Rule 4 . 
same color in the cut; a set of pairs of adjacent edges in the current cut for which Rule 1 is applicable; for each blue and each green vertex $v$ a counter for how many of its left-edges are not in the current cut, a second counter tallying in the current cut all occurrences of adjacent edge pairs in which exactly one edge is a left-edge of $v$ and a flag telling whether $v$ has been declared safe; finally, a set of vertices for which Rules 2 and 3 are applicable; this set contains all vertices for which the flag is on (i.e., the vertex is safe), and the two counters show 0 (all left-edges are in the current cut) and 2 (they are contiguous), respectively.

With this information the following type of algorithm can be performed efficiently: apply Rule 1 as long as possible, otherwise Rule 2 or 3. After applying one of these rule update the information outlined above appropriately and check whether Rule 4 or 5 is applicable. If so, apply it immediately. This can be done by a simple depth-first search.

We leave open further details of such an implementation. For the time analysis of this algorithm note first that with the information outlined above the respective applicability of Rules $1-5$ can be checked in constant time. An application of Rule 1 takes constant time. An application of Rule 2 or 3 takes time proportional to the degree of the vertex being swept over. Since an application of Rule 1 yields a vertex in the resulting subdivision $\mathscr{X}$ of constant degree (i.e., degree 4 ) we can say that any application of Rules 1-3 takes time proportional to the degree of the produces vertex of $\mathscr{X}$. The sum of the degrees of the vertices of $\mathscr{X}$ is $O(K)$ and thus all applications of Rules 1-3 together take time $O(K)$.

An application of Rule 4 takes time proportional to the number of blue edges traversed by the depth-first search. It is impossible to give a good bound for this number for an individual application of Rule 4. However, every blue edge can be traversed by such a depth-first search at most once. Thus all applications of Rule 4 together require $O(m)$ time. By the same argument all applications of Rule 5 together take $O(n)$ time. Thus we can summarize:

Lemma 2. For two convex subdivisions $\mathscr{B}$ and $\mathscr{G}$ of size $m$ and $n$, respectively, the convex subdivision $\mathscr{X}$ formed by the overlay of $\mathscr{B}$ and $\mathscr{G}$ can be constructed via a topological line sweep in optimal time $P(m+n+K)$, where $K$ is the size of $\mathscr{X}$.

It is worth pointing out that if we are only interested in enumerating the elements of the overlay subdivision $\mathscr{X}$, then our algorithm can do this using only $O(m+n)$ space.

The previous lemma might be considered a bit sweeping in that the nondegeneracy assumptions made at the beginning of this section have surreptitiously disappeared. We now want to show that these assumptions are sufficiently innocuous so that their omission in the statement of Lemma 2 is warranted.

Let us first state the possible degeneracies:

(i) A vertical edge is present.

(ii) A blue and a green vertex coincide.

(iii) A vertex lies on an edge of opposite color.

(iv) Two differently colored edges overlap in a line segment. 
One of the standard techniques of dealing with degeneracies is "perturbation." Conceptually, perturb the blue and the green subdivision such that no degeneracy occurs. Compute the overlay of the perturbed subdivisions, and finally remove from the resulting subdivision all degenerate parts that do not exist in the overlay of the unperturbed subdivisions, i.e., zero-length edges, zero-area regions, and identical vertices. Although this approach is correct and conceptually simple, we cannot afford it if we want to attain an $O(m+n+K)$ time algorithm, where $K$ is the true size of the unperturbed overlay, because perturbation can introduce too many extraneous elements. Consider for instance a blue vertex coinciding with a green vertex. None of their incident edges intersect in the unperturbed case. However, in the perturbed case, suddenly $O(b g)$ blue-green edge intersections can occur amongst these edges, where $b$ and $g$ are the degrees of the blue and green vertex, respectively. Moreover, if the incident edges are situated regularly about these two vertices, such an abundance of extraneous intersections arises for every possible perturbation.

Since the general perturbation approach that would collectively deal with all degeneracies proves to be too expensive we have to resort to addressing each type of degeneracy individually.

\section{(i) A Vertical Edge Is Present}

Vertical edges do not really constitute a degeneracy but rather an inconvenience for the formulation of the algorithm, which relies on such notions as a "left endpoint" or an edge lying "above a region." For every vertical edge $e$, regard its lower endpoint as its left endpoint and its upper endpoint as its right endpoint. If $e$ bounds a region $R$ from the left, regard $e$ as on top of $R$; if $e$ bounds $R$ from the right, take $e$ to be on the bottom of $R$. These conventions can be viewed as arising from a sufficiently small clockwise rotation of the entire subdivision.

\section{(ii) $A$ Blue Vertex $b$ and $a$ Green Vertex $g$ Coincide}

The combined vertex $z$ in the resulting subdivision $\mathscr{Z}$ can be swept over as soon as the union of the left-edges of $b$ and $g$ form a contiguous sequence of segments in the current cut. There is no need to check for safety. This observation suggests the following strategy: on discovering that $b$ and $g$ coincide (this occurs during checking for applicability of Rule 1), pair them up and create two counters for the pair analogous to the counters associated with plain vertices: one for the combined number of left-edges of $b$ and $g$ that are not in the current cut, the other one for the number of adjacent edge pairs in the current cut for which exactly one edge is not a left-edge of $b$ or $g$. When these counters show 0 and 2 , respectively, delete the duplicate left-edges of the joint vertex $z$ (see (iv)), and sweep over $z$, i.e., replace the sequence of left-edges of $b$ and $g$ in the current cut by the appropriately merged sequence of right-edges. 


\section{(iii) A Vertex $v$ Lies on an Edge e of Opposite Color}

On discovering this type of coincidence while checking for applicability of Rule 1 , split edge $e$ into two edges by introducing a new vertex $w$ that coincides with $v$. Thus this type of degeneracy is readily reduced to one of type (ii).

\section{(iv) Two Differently Colored Edges Overlap in a Line Segment s}

By virtue of the resolution of degeneracies of type (iii), the left and right endpoints of $s$, provided they exist, must be vertices arising from degeneracies of type (ii). Thus it only remains to ensure that $s$ is not recorded twice in the result subdivision. If $s$ has a right endpoint $z$, this is facilitated by the sweep step over $z$ as specified in the treatment of degeneracies of type (ii). If $s$ does not have a right endpoint, it must occur in the rightmost cut and this duplicate recording of $s$ can be readily removed from that cut and from the result subdivision after termination of the entire sweep.

The reader might ask whether Rules 4 and 5 need any amendments in order to deal with degeneracies. Strictly speaking this is not necessary. However, for implementation purposes the following should be noted. Using the terminology of Rule 4, the interesting case happens when $r$ is also the right endpoint of $e_{i}$ or $e_{k}$ (or both). In this case one has to make sure that the type of depth-first search described earlier for green vertices to be declared safe is started only through green left-edges of $r$ that lie between $e_{i}$ and $e_{k}$. Moreover, this has to be done irrespective of whether $r$ had been declared safe earlier.

This concludes our discussion of the handling of degenerate cases.

\section{Convolving Planar Tracings}

In [5] Guibas et al. introduced the notion of a tracing. They define a tracing to be a collection of states with signed multiplicities, where a state $s$ is a pair $(\dot{s}, \bar{s})$, with $\dot{s}$ a point in the plane and $\bar{s}$ a direction. A polygonal tracing is a tracing that can be partitioned into a finite number of subtracings which can only be of two types, a move or a turn. In a move $M$ the set $\{s \mid s \in M\}$ forms a (topologically) closed line segment $l$ in the plane, and $\{s \mid s \in M\}$ is a single direction which must be one of the two directions defined by the straight line containing $l$. In a turn $T$ the set $\{\dot{s} \mid s \in T\}$ is a singleton point, but the set $\{\bar{s} \mid s \in T\}$ is a connected and (topologically) closed interval of directions. Within each move or turn all states must have the same signed multiplicity, except for the two "endstates" which have only one-half of this multiplicity.

Intuitively, a move tells how often a car has passed through the states in the moves, where positive multiplicity means it traveled in direction $\bar{s}$ and negative multiplicity means it traveled in the direction opposite to $\bar{s}$ (but still facing in the direction $\bar{s}$ ). Similarly, a turn tells how often a car has turned over a certain 
interval of directions at point $\dot{s}$, where positive and negative multiplicities indicate left and right turns, respectively.

Using the sense of direction provided by the sign of the multiplicity one can define the notion of a balanced tracing. Intuitively, a tracing is balanced if every state was entered by a car as often as it was exited. This definition can be made precise using the concept of state counting functions. We refer the reader to [5]. We just note that since moves and turns by themselves are balanced except at their endstates, the notion of balance in the context of polygonal tracings just means that the moves and turns have to fit together in an appropriate way.

In [5] the concept of a convolution of two tracings is defined. If $a$ and $b$ are states in the tracings $A$ and $B$, respectively, such that $\bar{a}=\bar{b}=\phi$, then the state $c=(\dot{a}+\dot{b}, \phi)$ is in the convolution of $A$ and $B$. The multiplicity of $c$ is the product of the multiplicities of $a$ and $b$. This last statement has to be qualified though: the state $c$ in the convolution can arise simultaneously from several matching pairs of states in $A$ and $B$. In this case the multiplicity of $c$ is the sum of the product of the multiplicities of these matching pairs. Note that it can happen that the multiplicity of $c$ turns out to be 0 . In such a case we do not consider $c$ part of the convolution.

For polygonal tracings convolution can be viewed as a sum of convolutions of turns and moves, and turns and turns. The convolution of two turns $t_{1}$ and $t_{2}$ is a turn at position $\dot{t}_{1}+\dot{t}_{2}$. The set of directions swept by this turn is the intersection of the directions swept by $t_{1}$ and $t_{2}$ (in case this intersection is empty the convolution of the two turns is empty). The convolution of a turn $t$ and a move $m$ is empty if $\bar{m}$ is not in the set of directions swept by $t$, and otherwise it is a move with direction $\bar{m}$ over the line segment $\{\dot{x}+\dot{y} \mid x \in m, y \in t\}$. The multiplicities of the resulting moves and turns are again the product of the multiplicities of the pieces. We should also explain what we mean by "sum of convolutions of ...". Here sum means union, with the proviso that multiplicities are added.

The fact that states in the convolution of tracings $A$ and $B$ can simultaneously arise from several pairs of states from $A$ and $B$, so that one needs to worry about sums of multiplicities, and therefore about the possibility of their summing to 0 , creates difficulties for algorithms that compute the convolution of tracings. It is unclear how the output tracing of a convolution algorith should be represented. If no restrictions are put on the output representation, then computing the convolution essentially amounts to finding matching states. In such a case the continuity of the input tracings can be exploited in a nontrivial way. However, then the output might be presented in a rather redundant fashion. If, on the other hand, one requires the output tracing to be in a minimal, or canonical representation, the convolution algorithm has as to be able to deal with "chance" cancellations and coincidences which are difficult to infer just from the combinatorialgeometric structure of the input. We discuss this issue at some length in the appendix. Here we restrict our attention to tracings where these problems do not arise.

Call a balanced polygonal tracing simple if all states in the tracing have multiplicity +1 or -1 . A simple tracing is always a unique collection of "roundtrips," where a roundtrip is a cyclic sequence of alternating moves and turns 
with matching states at the transition points. This immediately yields a very natural and canonical representation for simple tracings: a collection of cyclic sequences. Call two simple tracings $A$ and $B$ transverse iff their convolution is simple and if no cancellations occur. A sufficient condition for the transversity of $A$ and $B$ is that no move of $A$ has the same direction as a move of $B$, and if $a_{1}, a_{2}$ and $b_{1}, b_{2}$ are the locations of turns in $A$ and $B$, respectively, then $a_{1}-a_{2} \neq b_{1}-b_{2}$. Note that in the space of pairs of all simple tracings, under any reasonable measure, the set of transverse pairs has measure one. Thus this class is quite natural and represents, in a sense, all inputs for a convolution algorithm that are in "nondegenerate" position.

Let the size of a simple tracing $A$ be the number of turns and moves in $A$. The size $K$ of the convolution $C$ of two simple transverse tracings $A$ and $B$ of size $m$ and $n$, respectively, can vary greatly. It can be as small as 0 , if no states in $A$ and $B$ have the same directional component, and it can be as large as $O(m n)$ (for an example see [5]). The running time of an efficient convolution algorithm should therefore depend not only on the size of the input but also on the size of the output. We outline an algorithm which is optimal in this respect.

Let $A$ and $B$ be two simple transverse tracings of size $m$ and $n$, respectively, and assume, for the time being, that both $A$ and $B$ consist of one roundtrip only. Constructing the convolution $C$ of $A$ and $B$ entails two steps. One has to discover all the moves and turns of $C$, and one needs to connect them into cyclic sequences that correctly reflect the roundtrips of $C$.

Using the result of Section 2 the first part is quite easy. To discover the turns of $C$ we have to determine which turns of $A$ and $B$ sweep over intervals of directions that overlap. To discover the moves of $C$ we have to determine the moves of $\boldsymbol{A}$ which have a direction that is contained in a directional interval swept over by a turn of $B$, and vice versa. Note that the direction of a move in a polygonal tracing is, for each turn incident to that move, an endpoint of the directional interval swept over by the turn. Thus the problem of finding all these intersections and containments among directions and intervals of cirections is really an instance of the problem of finding intersections between two contiguous sets of intervals, as presented in Section 2 . This means that we can find all moves and turns of $C$ in optimal time $O(m+n+K)$, where $K$ is the number of elements found.

It remains to show how the produced moves and turns of $C$ can be correctly hooked up into roundtrips. Without loss of generality assume that the moves and turns of $A$ were numbered from 0 to $m-1$ in the order in which they cyclically appear on the single roundtrip of $A$. Assume that the moves and turns of $B$ have been numbered similarly between 0 and $n-1$. Every move or turn of $C$ is the convolution of an element of $A$ and an element of $B$, and can thus be uniquely labeled by a pair $(i, j), 0 \leq i<m, 0 \leq j<n$. Note that for a turn $(i, j)$ of $C$, the moves that are incident to this turn can only have labels $(i-1, j),(i+1, j)$, $(i, j-1)$, or $(i, j+1)$. Simplicity of $A$ and $B$ ensures that exactly two of these moves will exist. It is not hard to see that after lexicographically sorting all these label pairs, and also lexicographically sorting the reversed pairs of labels, the two incident moves of every turn in $C$ can be found in linear time. Since the 
labels are integers in small ranges, this sorting can be done in time $O(m+n+K)$ using a standard radix sort.

Lemma 3. For two simple transverse tracings $A$ and $B$ of size $m$ and $n$, respectively, each consisting of one roundtrip, the convolution can be found in optimal time $O(m+n+K)$, where $K$ is the size of the tracing produced.

When $A$ and $B$ consist of more than one roundtrip, the following result holds:

Theorem 1. For two simple transverse tracings $A$ and $B$ of size $m$ and $n$, respectively, which together consist of $p$ roundtrips, the convolution can be found in optimal time $O(p \log p+m+n+K)$.

The $p \log p$ factor is used for determining which roundtrips of $A$ and $B$ have a nonempty convolution. It is possible to make a reduction argument from an element uniqueness problem to show that this $p \log p$ term is necessary and that therefore the stated time bound is indeed optimal. Finally, let us point out that the algorithm outlined above can be applied to nontransverse tracings and will yield a valid representation of the result. However, we can make no claim about optimality since we cannot guarantee that the produced representation is not redundant.

\section{Convolving Three-Dimensional Tracings}

When we attempt to extend our notion of tracings to dimensions higher than two, a number of modifications to the conceptual framework presented in [5] are indicated. First of all we must abandon the informal idea of "time" and car trips, since these are appropriate only to one-dimensional manifolds. We must replace the "open arrowheads" by a local notion of orientability, as is done in topology [8]. (Thus a tracing becomes a collection of orientable immersions of spheres.) In addition, the "solid arrowheads," or the way the car is facing, are most usefully thought of as normals to the manifold and not as tangents. The fundamental constraint on states then becomes that wherever the position manifold has a normal, the solid arrowhead of the corresponding state must be in the same, or the opposite, direction as that normal.

It would take us too far afield to try to make all these definitions precise here, so we will confine our attention to polyhedral tracings in three dimensions. While polygonal tracings in the plane are composed of two kinds of basic objects (moves and turns), polygonal tracings in space are composed of three fundamental components. The first is planar polygonal faces; in such a face the position component varies over some simple polygon while the direction component consistently coincides with one of the normals to the plane of the polyhedron. In our development here we will further assume that each such face is convex. The second type of component is a dihedral angle; such an angle corresponds to an edge of a polyhedron where the position of a state can vary along the edge, while the direction vector can be anywhere in an angle interpolating between the normals of two adjacent faces. Finally, the third component corresponds to a 
vertex of a polyhedron, where the position component is fixed while the direction component can be anywhere in a solid angle interpolating between the incident face normals. Again we will impose the condition that this solid angle of directions must be convex; in particular, this disallows saddle-points in the polyhedron.

Note that all fundamental components comprise two degrees of freedom: in face components these are both in the position of the state, in edge components one is in the position and one in the direction, and in vertex components both are in the direction. Just as in the planar case, we hook up such pieces to obtain immersions of spheres by observing the proper orientability constraints at the junctions so that the end result is an orientable manifold. For our purposes here we will confine our attention to an even more special kind of polyhedral tracing, what we call a monodromic tracing. Monodromic tracings in three dimensions are generalizations of monostrophic tracings in the plane. They are defined by the condition that for each direction there is exactly one state (taken with multiplicity \pm 1 ) that faces in this particular direction. Convex polyhedra can be viewed as monodromic tracings in an obvious way. A key property of monodromic tracings (and one we will heavily use in what follows) is that their direction maps, i.e., the assemblage of direction sets corresponding to each of their fundamental components, corresponds to a convex decomposition of the unit sphere.

The convolution of monodromic tracings can be defined in exact analogy with the corresponding planar concept. We match states from the factors having the same direction and put a result state in the convolution, facing in the common direction and positioned at the vector sum of the locations of the two factor states. It is easy to check that the convolution of monodromic tracings is again a monodromic tracing. In fact, for monodromic tracings that arise out of convex polyhedra (by taking consistently the, say, outward normal) the convolution will be another monodromic tracing which corresponds to the convex polyhedron associated with the Minkowski sum of the two factors. The direction map of such a convolution is of course again a convex subdivision of the sphere and, as we will see shortly, it is obtained from the two factor subdivisions by simply overlaying them.

The result of Section 3 can be used here to give us an optimal algorithm for convolving two monodromic three-dimensional tracings. The idea is very simple. Suppose we wish to convolve monodromic tracings $A$ and $B$. We can easily produce the direction maps $\mathscr{D}(A)$ and $\mathscr{D}(B)$ in linear time. (Although we have not discussed here in any detail the computer representation of monodromic tracings, a representation equivalent to two subdivisions-one recording the position component and the other the direction component of the tracing-is clearly possible, from any such representation the direction map is easily obtained). If the representation of $A$ has size $m$ and that of $B$ size $n$, then the corresponding direction maps can be computed and merged in time $O(m+n+K)$, where $K$ is the size of the overlayed map of $\mathscr{D}(A)$ and $\mathscr{D}(B)$. Once this overlayed map is available, the convolution $A * B$ can be immediately computed, since this map is the direction map for the convolution.

Why is this so? The faces of $A * B$ arise in one of three ways: (1) from a face of $A$ whose direction (normal) occurs in the solid angle of directions of a vertex 
of $B,(2)$ the same with $A$ and $B$ interchanged, and (3) from convolving two edges whose dihedral angles share a common direction (those are parallelogram faces). These three events are all recorded in the overlay of the direction maps and correspond respectively to (1) a vertex of $\mathscr{D}(A)$ lying in a face of $\mathscr{D}(B),(2)$ the same with $A$ and $B$ interchanged, and (3) the intersection of two edges, one from $\mathscr{D}(A)$ and one from $\mathscr{D}(B)$. Thus the vertices of the overlay direction map are in one-to-one correspondence with the faces of $A * B$. Similar arguments complete the proof. What this also shows is that the size of $A * B$ is $\Theta(K)$, and the full convolution can be computed in time $O(m+n+K)$. We have therefore shown that:

Theorem 2. The convolution $A * B$ of two monodromic tracings $A$ and $B$ is $a$ monodromic tracing and can be computed in time proportional to the size of its inputs plus the size of its output.

Such a result is clearly best possible. Note that the output size $K$ can vary from $\Theta(m+n)$ to $\Theta(m n)$. As a final point observe that the convolution of the monostrophic tracings stemming from two convex polytopes $P$ and $Q$ is the tracing corresponding to the Minkowski sum $P+Q$ (see [4]). Thus the last theorem implies that one can compute the Minkowski sum of two 3-polytopes in time proportional to the size of the inputs plus the size of the output.

\section{Appendix. What is Output Size?}

When talking and arguing about algorithms whose running time depends on the size of the input as well as the size of the output, certain subtleties arise that are usually irrelevant if the only complexity parameter is the input size. What is meant by "output size"?

As obvious as this question might be, it involves subtleties that hinge on the issue of representation. If one would like to make some sort of optimality claim for an algorithm, then "output size" must measure the intrinsic size of the mathematical object whose representation the algorithm is to produce. Output size cannot be the size of the representation itself, unless, of course, the produced representation is free of redundancies and its size truly reflects the size of the object represented. Preferably the representation should not just be irredundant but also canonical, in the sense that it is a unique denotation for the object in question.

For planar polygonal tracings such a canonical, irredundant representation might be as follows: set up a graph with vertices for the "transition" states between moves and turns, where edges with signed multiplicities correspond to either moves or turns interpolating between these states. If we require that no turns at a vertex overlap, and the same for moves on the same line, then we have a categorical representation except for full turns or full moves (in the 2SP sense, see [5]) since these have no distinguishable origin or destination state. Therefore such full turns and moves have to be represented specially. 
As desirable as it might be for an algorithm to produce canonical and irredundant representations, such a requirement also has certain drawbacks. It can happen that just verifying that some particular representation is indeed canonical can be more expensive than producing it. Thus it seems "unfair" to require an algorithm to convert its output into canonical form when it takes longer to perform this normalization than to generate a perfectly valid but noncanonical representation in the first place.

It is therefore tempting to drop the canonical form requirement and to allow a wider class of valid representations. For balanced polygonal tracings a natural class of representations are decompositions into "car trips," where each trip is represented as a circular alternating sequence of moves and turns properly joining together.

For the purpose of performing convolutions such a representation appears very convenient. The algorithm of Section 4 could be applied with hardly any change. However, a serious drawback of this approach is the fact that the produced representations might not only be noncanonical but also extremely redundant because of the cancellations possible in the calculus of tracing. In the case of convolutions of polygonal tracings one can come up with pathological examples where the output, when produced as a collection of car trips, will have size $\Theta(m n)$, whereas a canonical representation just has size $\Theta(m+n)$. These pathological cases are rather contrived and have the property that any small geometric perturbation of the input changes the combinatorial structure of the output (including its size) drastically. Analogous phenomena occur in algebraic manipulation problems.

This leaves the possibility that it might be unreasonable to measure the complexity of the problem of convolution just in terms of "input size" and "output size." It is conceivable that other parameters can be associated with pairs of tracings with which the complexity of convolution can be measured more naturally.

\section{References}

1. J. L. Bentley and T. A. Ottmann, Algorithm for reporting and counting gometric intersections, IEEE Trans. Comput. 28 (1979), 643-647.

2. B. Chazelle, Filtering search: a new approach to query-answering, SIAM J. Comput. 15 (1986), 703-724.

3. H. Edelsbrunner and L. J. Guibas, Topologically sweeping an arrangement, Proceedings of the 18th ACM Symposium on Theory of Computing, 389-403, 1986.

4. N. Grünbaum, Convex Polytopes, Interscience, New York, 1967.

5. L. J. Guibas, L. Ramshaw, and J. Stolf, A kinetic framework for computational geometry, Proceedings of the 24th IEEE Annual Symposium on Foundations of Computer Science, 100-111, 1983.

6. L. J. Guibas and J. Stolfi, Primitives for the manipulation of general subdivisions and the computation of Voronoi diagrams, ACM Trans. Graphics 4 (1985), 74-123.

7. J. Nievergelt and F. P. Preparata, Plane-sweep algorithms for intersecting geometric figures, Comm. ACM 25 (1982), 739-747.

8. E. H. Spanier, Algebraic Topology, Springer-Verlag, New York, 1966.

Received April, 1986, and in revised form October 30, 1986. 